Revista de Psicologia de la PUCP. Vol. XVII, 2, 1999.

\title{
COMPRENSIÓN DE LECTURA EN ALUMNOS DE CUARTO Y QUINTO GRADOS DE PRIMARIA DE LIMA
}

\author{
Silvia Morales Silva' \\ Pontificia Universidad Católica del Perú
}

Se evaluó 302 niños de cuarto y quinto de primaria con la prueba Evaluación de Inferencias y Meracognición (Morales, 1997). Se analizó (a) el grado de dificultad de las inferencias para cada grado, NSE y género: (b) la relación entre el grado, el NSE y el género con el puntaje en la prueba de comprensión $y$ con (c) el nivel de conciencia del propio rendimiento; y (d) la relación entre el nivel de conciencia del propio rendimiento y el puntaje. Los resultados indicaron que la dificultad de los tipos de inferencia fue similar para toda la muestra. Se comprobó la relación entre el puntaje en las tareas de comprensión con el grado y el NSE. Asimismo, se halló una relación entre la conciencia del propio desempeño en tareas inferenciales y el NSE. Finalmente, la asociación entre la conciencia del propio desempeño y el puntaje fue positiva, aunque de poca magnitud.

Palabras claves: comprensión, lectura, metacognición, inferencias, rendimiento, primaria.

Reading comprehension in fourth and fifth graders of Lima

The test of inferences and metacogtion (Morales, 1997) was used to assessed 302 fourth and fifth graders. It was analysed (a) the degree of difficulty of the inferences for each grade, SE level and gender; (b) the relation of grade, SE level and gender, with the score in the comprehension rask and with (c) the awareness about their achievement; and (d) the relation between the awareness about their achievement and the scores. The results showed that the degree of the inferences was similar for the whole sample. They also indicated a relation of the scores in the comprehension task with grade and the SE level. Likewise it was found a relation between the awareness of their achievement in the inferential task and the SE. level. The relation between the awareness of their achievement and the scores in comprehension was positive and weak.

Key words: comprehension, reading, metacognition, inferences, achievement, basic school.

1 Licenciada en Psicología Educacional de la Pontificia Universidad Católica del Perú. Actualmente es candidata al Diploma Europeo de Estudios Avanzados en la Educación del Talentoso (ECHA-KUN). Se desempeña como Jefe de Práctica en la PUCP y además se dedica al área de los problemas de aprendizaje y talento. Asimismo, ha investigado la comprensión de lectura y metacognición. Correo electrónico: e8712484@pucp.edu.pe. 

La lectura es una área importante en la investigación educacional actual. Entre otras cosas, la lectura es considerada una herramienta básica para el aprendizaje de todas las asignaturas en el contexto escolar. Como afirmaba Lerner (1993) en el mundo de hoy, a pesar de la variedad de medios de transmisión de información, no es posible mantenerse en el campo laboral o social sin saber leer. En este sentido, la presente investigación considera importante el estudio de todos los aspectos relacionados a la lectura comprensiva, especialmente la inferencia y la metacognición, y dentro del contexto peruano.

En la literatura psicoeducacional internacional se ha encontrado que la inferencia y la metacognición son elementos muy vinculados a la comprensión de lectura. En EUA, la metacognición se ha relacionado especialmente con el campo de los problemas especificos de aprendizaje y se ha dado aportes para la superación de dificultades en el campo de la lectura. En este ámbito, Schmitt (1990) construyó una prueba que evalúa el conocimiento del alumno sobre lo que debía hacer antes, durante y después de leer; Beal (1990b) investigó acerca de las habilidades de evaluación y revisión de los niños; asimismo, Alvermann, Bridge, Schmidt, Searfoss y Winograd (AV Manzo y UC Manzo, 1993) se interesaron por el conocimiento y la elección de estrategias esenciales para la comprensión. Y en el campo de las inferencias, Alliende (1982) ha planteado que la comprensión de lectura implica necesariamente inferir. Por otro lado, Carr, Dewitz y Parberg (1989) demostraron la efectividad del uso del método Cloze para el entrenamiento de comprensión inferencial con textos expositivos; mientras otros investigadores se interesaron en las inferencias de tipo causal en textos que son escuchados por niños y adultos (Ackerman, Silver y Glickman, 1990). 
En el Perú no se ha encontrado estudios acerca de la inferencia en la comprensión de lectura, siendo un tema tan importante en este campo. Por otro lado, recién se está empezando a investigar en el ámbito de la metacognición. Pinzás (1993) realizó un importante estudio, en el cual se examinó la inspección y regulación cognitivas en niños de los grados cuarto, quinto y sexto que pertenecian a tres tipos de colegios (estatal, privado pequeño y privado grande). Para ello utilizó la técnica de desorganización del texto (text disruption) y se basó en trabajos realizados por Markman (1977), Baker y Anderson (1981) y Zabrucky y Ratner en 1990 (Pinzás, 1993). Con respecto a la evaluación, en el contexto educativo, ha sido común medir la lectura utilizando materiales extranjeros (Alarcón, 1986); es decir, instrumentos creados para ser aplicados a personas que poseen otras características culturales, lingüísticas e incluso otro idioma. En ese sentido, el presente estudio considera importante el desarrollo de instrumentos de evaluación de la comprensión de lectura que, además de considerar las características particulares de algunas zonas de Lima, permitan comparar el nivel del niño con el de otros niños y obtener perfiles reales de los lectores. De esta manera, se contará con mayores elementos para desarrollar programas de enseñanza y mejoramiento de la lectura comprensiva.

\section{Preguntas de la investigación}

A partir de lo anteriormente expuesto, se propone investigar la evaluación de la comprensión de lectura en dos de sus aspectos más importantes: inferencias y metacognición. Se plantean las siguientes interrogantes: (a) ¿Existe un orden de dificultad de las cinco inferencias, diferente para cada grado escolar (cuarto y quinto), nivel socioeconómico (medio y bajo) y género?; (b) ¿El grado escolar, nivel socioeconómico y el género están relacionados con el rendimiento frente a cada uno de los tipos de inferencia y con (c) el nivel de conciencia relacionado a la calidad del propio desempeño? y (d) ¿Existe relación entre el nivel de conciencia sobre el propio desempeño y el rendimiento en las tareas inferenciales? 
Comprensión de lectura en alumnos de cuarto y quinto grados de primaria de lima

\section{Metodología}

En el presente estudio de nivel descriptivo y tipo causal compararivo se analizó los datos y se describió las relaciones que parecían existir entre las variables, sin llegar a explicar las causas de estas relaciones (Van Dalen y Meyer, 1991).

\section{Participantes}

Se trabajó con 302 niños de los grados cuarto y quinto de primaria que fueron elegidos a través de un muestreo no/probabilístico de tipo intencional. Ellos provenían de cuatro colegios de Lima, ubicados en zonas de los niveles socioeconómicos medio y bajo. En total fueron 160 varones y 142 mujeres. Como se puede apreciar se controló como variables independientes asignadas, el grado, el nivel socioeconómico y el género.

\section{Instrumento}

La prueba Evaluación de Inferencias y Metacognición (EIM), diseñada para fines de la investigación incluyó una sección para los datos personales, otra para la evaluación de las inferencias y una tercera para medir la metacognición.

La sección de Inferencia evaluó cinco tipos de ésta, que constituyeron las variables dependientes:

Inferencia de CausalEfecto. Se evaluará a través de una pregunta que pida al lector indagar sobre el motivo de alguna situación o efecto, con ayuda de las pistas que se encuentren en el texto, sin expresar ideas erróneas (falsas, inventadas o incompletas), y cuando su formulación incluya el interrogativo "¿Por qué...?”.

Inferencia de Caracteristicas. Se evaluará a través de una pregunta que pida al lector señalar alguna característica de algún personaje del texto relacionada a rasgos físicos, de carácter, estado de ánimto, gustos, 
apreciaciones personales, sentimientos, cualidades o defectos, con ayu$\mathrm{da}$ de las pistas que se encuentren en el texto, mediante una oración o frase que exprese tal característica, formulada con términos diferentes a los del texto, sin expresar ideas erróneas (falsas, inventadas o incompletas).

Inferencia de Léxico. Se evaluará a través de una pregunta que pida al lector señalar el significado de alguna palabra no conocida del texto con ayuda de las pistas que se encuentren en éste, mediante una oración o frase que pueda reemplazar a tal palabra sin alterar el significado y sentido del texto, y/o que esté relacionada con el significado del diccionario.

Inferencia Problema/Solución. Se evaluará a través de una pregunta que pida al lector que dé una posible solución a un problema, conflicto, inconveniente que se presente en el texto, con ayuda de las pistas que se encuentren en éste, sin expresar ideas erróneas (falsas, inventadas o incompletas).

Inferencia de la Idea Principal. Se evaluará a través de una pregunta que pida al lector colocar un título al texto con ayuda de las pistas que se encuentren en éste, mediante una oración o frase que sintetice y englobe todos los hechos ocurridos en la historia, sin expresar ideas erróneas (falsas, inventadas o incompletas).

Asimismo se consideró como variable dependiente el nivel de conciencia sobre la calidad del propio desempeño.

Se refiere al grado de certeza de la estimación que da el alumno sobre la calidad de su propio desempeño ante tareas de comprensión inferencial, al compararla con su desempeño real. Es decir, en qué medida el alumno se da cuenta de la calidad real de su rendimiento o de cuánto realmente ha entendido del texto.

El evaluado debía leer cada texto de aproximadamente un párrafo de extensión y luego responder las preguntas que correspondían a los 
Comprensión de lectura en alumnos de cuarro y quinto grados de primaria de Lima

cinco tipos de inferencia en formato de opción múltiple con tres opciones. Los temas tratados estuvieron relacionados con vivencias de los niños de las edades evaluadas. Asimismo, no se colocó título a los cuentos para no interferir en o sesgar la respuesta sobre la idea principal.

La sección de Metacognición incluyó los siguientes ítemes:

- Ahora vas a pensar en cómo has respondido a las preguntas anteriores.

- ¿Cuál de los textos crees que has comprendido y respondido mejor?

- ¿Cuál de los textos crees que no has comprendido ni respondido tan bien?

Los pasos que se siguieron para la construcción del instrumento fueron:

1. Después de revisar bibliografía y consultar con investigadores sobre inferencias, metacognición, construcción de pruebas y estadística, se contactó cona la muestra, redactándose una lista de términos a los que ésta es expuesta y otra lista de los términos usados por los niños (como lo hiciera Aspíllaga en 1990).

2. Redacción de la prueba.- Se redactó ocho textos con preguntas que fueron revisados por cuatro psicólogas, una profesora de primaria, un experto en redacción y linguística y un experto en construcción de pruebas. El coeficiente $\mathrm{V}$ de Aiken fue de $93 \%$.

3. Evaluación de la prueba en proceso.- Se evaluó mediante la prueba con preguntas abiertas a ocho niños de cada sector de la muestra. Luego se redactó las opciones.

4. Evaluación etapa 1 con EIM 1.- Con la prueba lista (ocho subpruebas y opciones), se evaluó 204 estudiantes para escoger grado y las cinco mejores subpruebas. Se realizó en dos sesiones, cuatro subpruebas por día. Había dos versiones de la prueba, cada una para cada mitad de la muestra: ab empezó con las subpruebas $1 \mathrm{~A}, 2 \mathrm{~A}, 3 \mathrm{~A}$ y $4 \mathrm{~A}$ y 
terminó en la segunda sesión con $1 \mathrm{~B}, 2 \mathrm{~B}, 3 \mathrm{~B}$ y 4B; la versión ba fue a la inversa.

5. Análisis de resultados.- Se analizó la confiabilidad (K-R 20), nivel de dificultad, correlación subprueba/prueba total. El grado al que más se adecuaba la prueba fue cuarto y las cinco subpruebas más confiables que conformaron EIM 2 fueron $2 \mathrm{~A}, 3 \mathrm{~A}, 1 \mathrm{~B}, 3 \mathrm{~B}$ y $4 \mathrm{~B}$.

6. Evaluación Etapa 2 con EIM 2.- Se evaluó a 98 niños de cuarto grado -requeridos para los análisis estadísticos- con EIM2. Luego se analizó la confiabilidad.

7. Redacción de la prueba final EIM3. - Se reordenó las subpruebas e ítemes según su dificultad, se determinó los ejemplos de explicación y se rediseñó la hoja de protocolo.

Validez de Contenido de la sección de inferencias.- Teniendo como dominio de contenido los principales tipos de inferencias (Asociación Internacional de Lectura,1990; Montes y Gonzáles, 1982), se eligió una muestra representativa, se dio el peso correspondiente a cada una de las categorías en que se dividió el dominio de contenido y se obtuvo los tipos de inferencias. Los jueces estuvieron de acuerdo en que los ítemes -después de algunas modificaciones sugeridas- representaban a tales tipos de inferencias. Ellos asignaron a cada reactivo una categoría que se comparó con la de los constructores de la prueba. De lo anterior resultó un acuerdo de $93 \%$ mediante el coeficiente $\mathrm{V}$ de Aiken.

Validez de Contenido de la sección de Metacognición. Para esta segunda área se tomó como dominio de contenido los tipos de pregunta basados en los planteamientos de AV Manzo y UC Manzo (1993), que evaluaban el autoconocimiento, el conocimiento de la tarea y el automonitoreo, las pruebas que evaluaban las estrategias de procesamiento (autorreporte y predicciones sobre el propio desempeño) y la inspección y regulación cognitiva propuesta por Pinzás (1993) (autocuestionamiento, confirmación de suposiciones y formulación de predicciones). Luego de la consulta con especialistas, se eligió la zona 
del dominio a evaluar, de manera intencionada. De esto resultó una única pregunta que se visualizó como un paso importante para el estudio de la metacognición de los niños limeños.

Confiabilidad de EIM.- Se utilizó la fórmula Kuder Richardson 20 por tratarse de ítemes dicotómicos con diferente nivel de dificultad; adicionalmente, se analizó el nivel de dificultad y la correlación ítem/ test. Se consideró como valor adecuado de confiabilidad el coeficiente mayor o igual a .70 para la prueba total (Guilford, 1956 y Nunnally, 1978 , en Kline, 1994, para una prueba de habilidades con un mínimo de diez ítemes) y de .4 para cada subprueba por separado. Además, el nivel de dificultad promedio debía ser cercano a .50 (Anastasi, 1980) y el valor de correlación ítem/test debía ser mayor a .20 (Brown, 1980).

EIM1. Se obtuvo un índice de confiabilidad de la prueba total de .81 , tanto para cuarto como para quinto grado. $Y$ dado que el índice de dificultad de la prueba para quinto grado (.71) fue mucho más alto que para cuarto grado (.65), se decidió - de acuerdo a los objetivos de la prueba- que ésta sea destinada para cuarto grado.

EIM2. Al evaluar a 98 niños más del grado elegido, se obtuvo confrabilidad de la prueba .75 (cuarto .72 y quinto .78).

\section{Procedimiento}

Después de realizar el contacto con los directores de los colegios seleccionados, se observó clases y se llevó a cabo la primera parte de la evaluación en dos días seguidos. Luego se procesó y analizó los datos. De esta manera se eligió el grado con el que la prueba demostró tener la mayor confrabilidad y validez (cuarto) y las cinco subpruebas con las confiabilidades más altas que conformarían EIM2. Finalmente se realizó la segunda etapa de evaluación con niños del grado elegido mediante EIM2. Así se dererminó, con una muestra mayor, las caracrerísticas psicométricas de la prueba y los posteriores análisis de manera más precisa. De estos resultados se elaboró el diseño final de la prueba y la discusión y conclusiones acerca de las preguntas planteadas. 


\section{Resultados}

De acuerdo a los objetivos y a las preguntas de investigación se obtuvo que la prueba EIM obtuvo validez y confiabilidad (EIMl=.81 y EIM2 =.75) para cuarto y quinto grado de colegios estatales de Breña y particulares de La Molina, con mayor capacidad de discriminación entre puntajes en el grado menor. En relación a los puntajes obtenidos con EIM, éstos fueron fueron significativamente mayores para el quinto grado y el estrato medio en comparación con el cuarto grado y el nivel bajo, $y$ no hubo diferencia significativa entre los varones y las niñas.

Por otro lado, el orden de dificultad de los tipos de inferencia fue similar para ambos grados, niveles socioeconómicos y géneros estudiados. Y se comprobó la relación entre el rendimiento frente a cada uno de los tipos de inferencia -excepto Léxico-con el grado y con el nivel socioeconómico, mas no con el género -excepto Léxico, que fue más difícil para las niñas (Cuadros 1, 2 y 3).

\section{Cuadro 1}

Análisis de Varianza según Grado, Nivel Socioeconómico y Género para los tipos de Inferencia

\begin{tabular}{|c|c|c|c|c|c|c|}
\hline Puente de valiador & 8 & Cofrist & Craracter & $12 \times \mathrm{ico}$ & Problenut & $\begin{array}{l}\text { Tder } \\
\text { Principal? }\end{array}$ \\
\hline $\begin{array}{l}\text { Grado de } \\
\text { estudios (Gr) }\end{array}$ & 1 & $8.38^{* *}$ & $3.90^{*}$ & 3.15 & $15.05^{* * * *}$ & $4.25^{\star}$ \\
\hline NSE (N) & 1 & $36.28^{* * * *}$ & $35.89^{* * * *}$ & $41.66^{* * * * *}$ & $20.95^{* * * *}$ & $23.06^{* * * *}$ \\
\hline Género (Ge) & 1 & $6.20^{*}$ & 1.51 & 2.87 & 1.79 & .00 \\
\hline $\operatorname{GrxN}$ & 1 & $5.11^{*}$ & .75 & .49 & .08 & .32 \\
\hline GrxGie & 1 & 1.69 & .26 & .16 & .10 & .28 \\
\hline GexN & 1 & $5.84^{*}$ & 1.94 & .46 & .01 & .34 \\
\hline GexNxGr & 1 & 3.62 & 3.37 & .68 & 1.22 & .11 \\
\hline
\end{tabular}

${ }^{*} p<.05{ }^{* *} p<.01{ }^{* * * *} p<.0001$ 
Comprensión de lectura en alumnos de cuarto y quinto grados de primaria de Lima

Cuadro 2

Análisis de Varianza según Grado y Género para la Inferencia Causa/Efecto

\begin{tabular}{l|c|c|}
\hline Fuente de variación & \multicolumn{3}{|c|}{ CausalEf: } \\
\hline & gl & F \\
\hline Grado (Gr) & 1 & 8.92 \\
Género (Ge) & 1 & .18 \\
Gr X Ge & 1 & $4.91^{*}$ \\
\hline${ }^{*} p<.05$ & & \\
\hline
\end{tabular}

Cuadro 3

t-Student según Género para dos Tipos de Inferencia

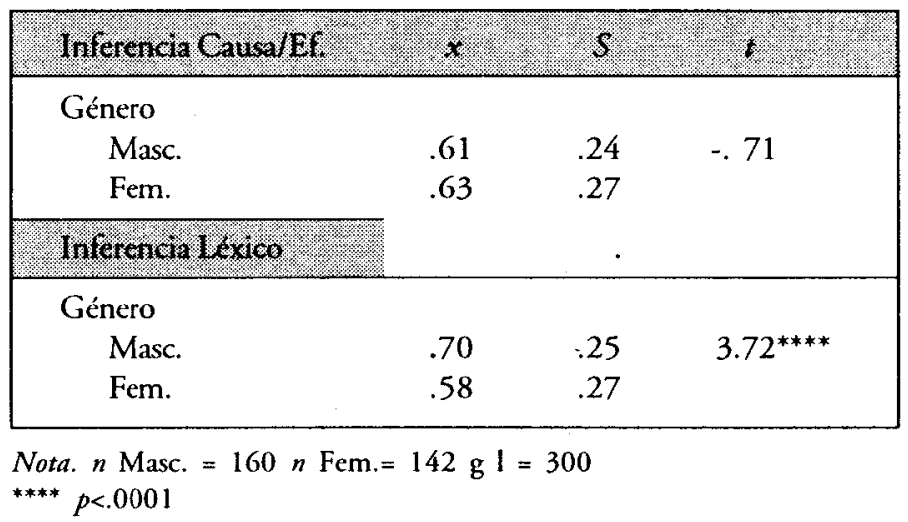

Se encontró relación de la conciencia de la calidad del propio desempeño en tareas inferenciales con el nivel socioeconómico, mas no con las variables grado escolar ni género (Cuadro 4). Y la asociación entre la conciencia del propio desempeño frente a tareas inferenciales y el rendimiento real frente a este tipo de tareas fue positiva y débil $\left(r_{\mathrm{s}}=.12\right)$. El Cuadro 5 amplía la información. 


\section{Discusión y Recomendaciones}

En cuanto a los puntajes, la ventaja en las puntuaciones del quinto grado, el estrato medio y los varones en comparación con el cuarto grado, el nivel bajo y las niñas coinciden con otros estudios realizados en nuestro medio. En 1988, Majluf (Majluf, 1993), quien utilizó la prueba de De la Cruz en Lima y otras ciudades del Perú, encontró un efecto de la estimulación y del nivel sociocultural en el desempeño lector. Además descubrió que en la costa y sierra los niños puntuaban más alto que las niñas. Con la misma prueba, Vega en 1988 (Thorne, 1991) obtuvo puntajes más altos en colegios privados de Lima, en comparación con los estatales.

Por otro lado, Thorne (1991) evidenció, al evaluar con su Prueba de Comprensión de Lectura Inicial a niños de primer grado, que los alumnos de estratos medios y altos puntuaban más que los de estratos

Cuadro 4

Análisis Chi Cuadrado de Asociación de Efectividad en la Predicción con Grado, Nivel Socioeconómico y Género

\begin{tabular}{|c|c|c|c|c|c|c|}
\hline \multirow{2}{*}{ or mediv } & \multicolumn{2}{|c|}{ Crato } & \multicolumn{2}{|c|}{ NSE } & \multicolumn{2}{|c|}{ dentero } \\
\hline & $4^{\circ}$ & $5^{\circ}$ & Medio & Bajo & Mase. & Fen. \\
\hline 0 & 19.0 & 18.6 & 12.2 & 25.3 & 15.6 & 22.5 \\
\hline 25 & 14.0 & 25.5 & 15.5 & 20.1 & 17.5 & 18.3 \\
\hline 50 & 33.5 & 30.4 & 35.8 & 29.2 & 34.4 & 30.3 \\
\hline 75 & 16.5 & 15.7 & 14.9 & 17.5 & 16.3 & 16.2 \\
\hline 100 & 17.0 & 9.8 & 21.6 & 7.8 & 16.3 & 12.7 \\
\hline Total & 100 & 100 & 100 & 100 & 100 & 100 \\
\hline Pearson & \multicolumn{2}{|c|}{7.62} & \multicolumn{2}{|c|}{$19.06^{* * *}$} & \multicolumn{2}{|c|}{2.98} \\
\hline Cramer & \multicolumn{2}{|c|}{.--} & \multicolumn{2}{|c|}{$.25^{* * *}$} & \multicolumn{2}{|c|}{.--} \\
\hline
\end{tabular}

Nota. $\mathrm{g} \mathrm{l}=4$ en todos los casos. ${ }^{* * *} p<.001$ 
Comprensión de lectura en alumnos de cuarto y quinto grados de primaria de lima

Cuadro 5

Puntajes Correspondicntes a Cada Tipo de Predicción

\begin{tabular}{|c|c|c|c|c|c|c|c|c|}
\hline 1.:. & 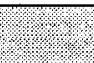 & \%. Elec & vididien & Predicaion & & Total & F ratio & f prob \\
\hline & 0 & 25 & 50 & 75 & 100 & & & \\
\hline$X$ & 15.46 & 15.81 & 16.04 & 16.29 & 16.80 & 16.04 & .86 & .49 \\
\hline$S$ & 3.66 & 3.65 & 3.64 & 4.36 & 4.05 & 3.82 & & \\
\hline & $(n=57)$ & $(n=54)$ & $(n=98)$ & $(n=49)$ & $(n=44)$ & $(n=302)$ & & \\
\hline
\end{tabular}

bajos. Asimismo, las niñas tuvieron mejores puntajes en niveles medios, mientras que los varones de los colegios estatales aventajaron a las mujeres.

Pérez y Vizurraga (1989), por su parte, con la Prueba de Comprensión de Lectura para niños de tercer y cuarto grado, encontraron diferencias significativas entre grados; pero, contrariamente a otras investigaciones, los mejores resultados fueron del grado menor. En cuanto al género, los puntajes favorecieron a los niños.

La semejanza en el orden de dificultad de las inferencias para cada grado, nivel socioeconómico y género que se encuentra en la presente investigación puede deberse a que la secuencia de adquisición de conocimiento presenta la misma estructura para todo individuo. Y la diferencia estaría en el nivel de desarrollo alcanzado, como afirmó Blumen (1997) en un estudio sobre las teorías del desarrollo cognitivo. Estos resultados coinciden con una investigación de Silverstein, Morita y Belger (1983) quienes al evaluar con la Prueba de Conceptos Básicos de Boehm (B'BC) a niños de Kindergarten encontraron que los íremes mostraron el mismo orden relativo de dificultad para ambos géneros y para niños de diferente nivel socioeconómico.

Sin embargo, los resultados no convergen con otros autores como Fischer y Klahr (Blumen, 1997), quienes afirmaron que el curso del 
desarrollo puede diferir de un niño a otro, a pesar de que los mecanismos a la base del cambio cognitivo son los mismos para todos los niños.

La comprobada relación que se da entre el rendimiento frente a los tipos de inferencia con el grado escolar y con el nivel socioeconómico, mas no con el género se encuentra en la línea de los estudios de Vygotsky (1978) y Sternberg (1985) (Blumen, 1997) quienes revelaron el rol crucial del medio ambiente (social y cultural) en la cognición. Esto se muestra con el estudio de Silverstein et al. (1983), donde el puntaje promedio se elevaba considerablemente del nivel bajo al alto. En el medio peruano, Majluf (1993) ha evidenciado en localidades de la costa, sierra y selva, que la estimulación ambiental y el estado nutricional afectan el rendimiento cognitivo con la consecuente desventaja para los niños de nivel bajo.

Las diferencias de puntajes por grados se pueden traducir a diferencias por edades. En este sentido, algunos investigadores también han encontrado efectos de la cdad sobre el rendimiento. Por ejemplo, los teóricos de los estadios (Blumen) han señalado limitaciones relacionadas con la edad. Ellos afirmaron que las limitaciones disminuyen a medida que el niño crece, incrementándose las capacidades. Estos resultados coinciden con Ackerman et al. (1990), quienes encontraron en sus estudios sobre inferencias causales que la probabilidad para resolver inferencias se incrementaba con el grado, debido a diferencias evolutivas en el conocimiento de base y en la extensión de la memoria de trabajo y a la poca habilidad para entender relaciones lógicas entre oraciones y eventos e integración de diferentes oraciones. Y. en 1984, Rosales (Pinzás, 1993), evidenció el progreso del vocabulario en la comprensión de lectura según el grado, en un estudio con niños de los grados cuarto a sexto.

La mayor habilidad en los varones para resolver inferencias de léxico coincide con los resultados de Majluf (1993), quien ha encontrado una ventaja del varón respecto a la mujer, aunque ésta se ha dado a nivel 
general. Sin embargo, estos resultados no coinciden con los de Stanley y Benbow (Moir y Jessel, 1991) o los de Lerner (1993), quienes sostuvieron que las niñas muestran mayor capacidad verbal en los primeros años del colegio y que existe mayor probabilidad de que los varones tengan problemas de lectura. Asimismo, tampoco se asemeja a lo que obtuvieron Silverstein et al. (1983), quienes evaluaron con el BTBC a niños de Kindergarten, y no encontraron diferencias significativas según género.

En general, el orden similar de dificultad de las inferencias sugiere que la enseñanza de la comprensión de lectura relacionada a estos cinco tipos de inferencia cuente con la misma secuencia para cada grado, nivel socioeconómico y género. Además, que la enseñanza empiece por los tipos de inferencias más fáciles y continúe con los más difíciles; de este modo iría de lo que está más relacionado al niño a lo más complejo para que el aprendizaje resulte efectivo (Giroux, 1988).

Por otro lado, los resultados sugieren que el nivel de dificultad varíe de cuarto a quinto grado y del nivel socioeconómico bajo al medio. En este sentido se podría considerar el reforzamiento desde los primeros años escolares de la base lingüística en el estrato bajo para poder mejorar su desempeńo. Esto, considerando que la expansión del vocabulario es un medio efectivo para mejorar la comprensión de lectura (Lerner, 1993). En cuanto al género, los resultados coinciden en que éste no será motivo para hacer diferencias en la enseñanza de los tipos de inferencia, excepto la de Léxico que implicaría un mayor reforzamiento conceptual para las niñas.

En relación al nivel de conciencia del propio desempeño, en el nivel socioeconómico medio existen más nińos que presentan un conocimiento real sobre su rendimiento; lo cual puede deberse a que en sus centros educativos la enseñanza suele ser más flexible y se incentiva la expresión de opiniones $y$ el debate en grupo para tomar decisiones. De manera contraria, en colegios del estrato bajo, la instrucción conserva características tradicionales, donde el alumno tiende a ser más pasivo (Giroux, 1988; 
Brophy y Good, 1991). Si no es capaz de opinar acerca de un tema, entonces no se acostumbra a evaluar o criticar a otros y por lo tanto, tampoco logrará el paso más complejo: evaluarse a sí mismo; como afirmó Markman en 1977 (Pinzás, 1993), a lo largo del desarrollo se va tomando un rol activo o autodirigido en ciertas áreas de la cognición, pero si éstas no se estimulan, no se desarrollarán efectivamente.

Estos resultados están en la línea de Pinzás (1993), quien en su estudio sobre inspección y regulación cognitiva en los grados cuarto, quinto y sexto de colegios estatales y privados, evidenció que los niños de mayor habilidad metacognitiva fueron los de colegios privados, en comparación con los de colegios estatales.

Por otro lado, no se encuentra diferencias significativas por grado en relación a la conciencia del desempeño. Es posible que las hubiera si los grados estuvieran más diferenciados. En numerosos estudios (Beal, 1990a, 1990b; Tobias, 1995) se ha comprobado que a medida que los niños crecen, se vuelven más expertos, y dedican mayor tiempo al procesamiento en la planificación de la solución de un problema que a la solución del mismo.

La débil correlación entre la conciencia de la efectividad del propio desempeño con el puntaje de la prueba coincide con los estudios de Körkel y Schneider (1991) y Ehrlich (1991). Ellos explicaron que el llamado conocimiento metacognitivo declarativo (CMD) sólo juega un rol pequeño como predictor de aquello que se va a recuperar, contrariamente al conocimiento metacognitivo de procedimiento (CMP) que sí es un predictor importante. Esto significa que el lector puede aprender a utilizar estrategias de comprensión efectivamente de modo automático, pero si no es consciente de su procesamiento de información, poseerá un limitado conocimiento metacognitivo declarativo. Ehrlich (1991) tampoco ha encontrado relación entre la autoevaluación de la comprensión y los índices objetivos de la comprensión. Asimismo, Mateos y Alonso (1991) han encontrado relación entre la autorregulación -y no el CMD que es evaluado con EIM- y la comprensión. 
Comprensión de lectura en alumnos de cuarto y quinto grados de primaria de Lima

Por otro lado, la relativa baja relación entre el nivel de conciencia del propio desempeño y el puntaje de la prueba, puede deberse a la llamada ilusión de la comprensión. Esto significaría que un grupo de los niños no notó que su comprensión era pobre, porque construyó una representación mental que tenía coherencia a nivel local, sin embargo la representación de alto orden falló (Ehrlich, 1991).

Estos resultados sugieren la importancia de crear espacios donde se incentive la crítica y reflexión, sobre todo en el nivel socioeconómico bajo, donde el contexto sociocultural refuerza lo que podríamos denominar la pasividad cognitiva de los estudiantes. Asimismo se indica que se debe elevar el CMP de los estudiantes para incrementar su rendimiento en tareas de comprensión de lectura; ésto, sin dejar de incentivar el CMD.

En relación a la prueba EIM se sugiere aplicarla a una muestra más representativa de la ciudad de Lima y obtener sus características psicométricas. Asimismo, extender la prueba a otros tipos de inferencia. Por otro lado sería interesante revisar el contenido de los textos de la prueba de tal manera que se mantenga un balance entre los temas masculinos y femeninos y así controlar la motivación hacia la lectura. Asimismo, se sugiere investigar la comprensión de las inferencias relacionada al conocimiento previo y relacionada al formato de la lectura.

\section{Conclusiones}

- La prueba EIM cuenta con validez y confrabilidad para cuarto y quinto grado de colegios estatales de Breña y particulares de La Molina.

- Los puntajes de la prueba EIM son significativamente mayores para el quinto grado y el estrato medio en comparación con el cuarto grado y el nivel bajo.

- No existe diferencia significativa entre los varones y las niñas.

- Se presenta un orden de dificultad de los tipos de inferencia similar 
para ambos grados, niveles socioeconómicos y géneros estudiados.

- Se comprueba la relación entre el rendimiento frente a cada uno de los tipos de inferencia con el grado (a excepción de Léxico) y con el nivel socioeconómico, mas no con el género (a excepción de Léxico).

- Existe relación de la conciencia de la calidad del propio desempeño en tareas inferenciales con el nivel socioeconómico, mas no con las variables grado escolar ni género. Y la asociación entre la conciencia del propio desempeño frente a tareas inferenciales y el rendimiento real frente a este tipo de tareas es positiva y débil.

- Se sugiere considerar los resultados encontrados al diseñar un programa de comprensión de lectura que focalice las inferencias; es decir, seguir la misma secuencia para cada grado, nivel socioeconómico y género e ir del tipo de inferencia más fácil hasta el más difícil. Y el nivel de dificultad deberá variar de cuarto a quinto grado y del nivel socioeconómico bajo al medio. Asimismo, reforzar la base lingüística y de autorreflexión desde los primeros años escolares en el estrato bajo.

\section{Referencias}

Ackerman, B., Silver, D. y Glickman, I. (1990). Concept availability in the causal inferences of childrens and adults. Child Development, 61(1), 230- 246.

Alarcón, R. (1986). Psicologia, pobreza y subdesarrollo. Lima: INIDE. Alliende, F. (1982). La comprensión de la lectura y su desafío. Lectura y Vida, 1, 4-22.

Anastasi, A. (1980). Tests psicológicos. Madrid: Aguilar.

APOYO-Opinión y Mercado (1995). Niveles socioeconómicos 1995:

Lima Metropolitana. Lima: APOYO-Opinión y Mercado.

Asociación Internacional de Lectura (1990). Nuevas perspectivas en la enseñanza de la lectura. Lectura y Vida, 1, 34-36. 
Comprensión de lectura en alumnos de cuarto y quinto grados de primaria de Lima

Aspíllaga, S. (1990). Propuesta de un instrumento para el estudio del vocabulario infantil. Tesis de bachiller en psicología, Pontificia Universidad Católica del Perú, Lima, Perú.

Beal, C. R. (1990a). Development of knowledge about the role of inference in text comprehension. Child Development, 61, 10111023.

Beal, C. R. (1990b). The development of text evaluation and revision skills. Child Development, 61(1), 247-258.

Blumen, S. (1997). El desarrollo de las habilidades cognitivas según los avances en las teorías psicológicas. Revista de Psicología, 15(1), 54-95.

Brophy, J.E. y Good, T.L. (1991). Looking in classrooms. Nueva York: Harper Collins.

Brown, F. (1980). Principios de la medición en psicologia y educación. México: Manual Moderno.

Carr, E., Dewitz, P. y Patberg, J. (1989). Using cloze for inference training with expository text. The Reading Teacher, 42(6), 380385.

Ehrlich, M-F. (1991). Metacognition and reading comprehension: Theoretical and methodological problems. En M. Carretero, M. Pope, R-J Simons y J. Pozo (Eds.), Learning and Instruction (pp. 351-363). Gran Bretaña: BPCC Wheatons.

Giroux, H. (1988). Schooling and the struggle for public life. Mineapolis: University of Minnesota Press.

Kline, P. (1994). The handbook of psychological testing. Nueva York: Routledge.

Körkel J. y Schneider, W. (1991). Domain-Specific versus metacognitive knowledge effects on text recall and comprehension. En $M$. Carretero, M. Pope, R-J Simons y J. Pozo (Eds.), Learning and Instruction (pp. 311-323). Gran Bretaña: BPCC Wheatons.

Lerner, J. (1993) Learning disabilities. Theories, diagnosis and teaching strategies ( 6 ed). Boston: Houghton Mifflin Company.

Majluf, A. (1993). Marginalidad, inteligencia y rendimiento escolar. Lima: Brandon Enterprise. 
Manzo, A. V. y Manzo, U. C. (1993). Literacy disorders holistic diagnosis and remediation. Orlando: Harcourr Brace Jovanovich .

Mateos, M. y Alonso, J. (1991). Metacognition and reading comprehension: Strategies for comprehension monitoring training. En M. Carretero, M. Pope, R.-J. Simons y J. Pozo (Eds.), Learning and Instruction (pp. 273-292). Gran Bretaña: BPCC Wheatons.

Moir, A. y Jessel, D. (1991). El sexo en el cerebro: La verdadera diferencia entre hombres y mujeres. Barcelona: Planeta.

Montes de Gonzáles, M. y Gonzáles de Mira, C. (1982). Inventario informal de lectura (IIL). Lectura y Vida, 4, 4-14.

Morales, S. (1997). Comprensión de lectura en niños de los grados cuarto y quinto de primaria de Lima: Análisis de inferencias y metacognición. Tesis de licenciatura en psicología, Pontificia Universidad Católica del Perú, Lima.

Pérez, M. y Vizurraga, S. (1989). Estrategias para el desarrollo de la comprensión de lectura para el tercer y cuarto grado de educación primaria. Memoria de Bachiller en Educación, Pontificia Universidad Católica del Perú, Lima, Perú.

Pinzás, J. (1993). Cognitive monitoring in reading comprehension: a study of differences among schools in Lima. Nimega: Quickprint.

Schmitt, M C. (1990). A questionnaire to measure children's awareness of strategic reading processes. The reading Teacher Journal of the IRA, 43(7), 454-461.

Silverstein, A.B., Morita D.N. y Belger, K.A. (1983). Sex differences and sex bias on the Boehm Test of Basic Concepts: Do they exist? Psychology in the Schools, 20, 269-271.

Thorne, C. (1991). A study of beginning reading in Lima. Nimega: Quickprint.

Tobias, S. (1995). Interest and metacognitive word knowledge. Journal of Educational Psychology, 87(3), 399-405.

Van Dalen, D.B. y Meyer, W. I. (1991). Manual de técnica de la investigación educacional. México: Paidós. 